\title{
Severe infections caused by multidrug-resistant non-fermentative bacilli in southern Poland
}

\author{
Agnieszka Chmielarczyk $k^{1, A-D, F}$, Monika Pobiega ${ }^{1, B, C}$, Grzegorz Ziółkowski2, ${ }^{2}$, Monika Pomorska-Wesołowska, ${ }^{3, B}$, \\ Dorota Romaniszyn ${ }^{1, C}$, Lech Krawczyk ${ }^{2, E}$, Jadwiga Wójkowska-Mach ${ }^{1, A, E, F}$ \\ 1 Chair of Microbiology, Jagiellonian University Medical College, Kraków, Poland \\ ${ }^{2}$ Higher School of Medicine in Sosnowiec, Poland \\ ${ }^{3}$ Department of Microbiology, Analytical and Microbiological Laboratory, KORLAB Non-public Healthcare Center, Ruda Śląska, Poland \\ A - research concept and design; $\mathrm{B}$ - collection and/or assembly of data; $\mathrm{C}$ - data analysis and interpretation; \\ $D$ - writing the article; $E$ - critical revision of the article; $F$ - final approval of the article
}

\section{Address for correspondence \\ Agnieszka Chmielarczyk}

E-mail: aga.chmielarczyk@gmail.com

\section{Funding sources}

This work was supported by a grant from the National Science Center (No. DEC-2012/05/B/ NZ7/02880). The sponsor provided the funding for the project only.

\section{Conflict of interest}

None declared

\section{Acknowledgements}

The work presented here was carried out in a collaboration between all authors. All authors have seen and approved the manuscript. Some of the results shown in this publication were presented as a poster at the $9^{\text {th }}$ Healthcare Infection Society Conference: November 16-18, 2014, Lyon, France.

Received on May 2, 2016

Reviewed on 0ctober 10, 2016

Accepted on January 17, 2017

DOI

$10.17219 /$ acem $/ 68545$

\section{Copyright}

Copyright by Author(s)

This is an article distributed under the terms of the

Creative Commons Attribution Non-Commercial License

(http://creativecommons.org/licenses/by-nc-nd/4.0/)

\begin{abstract}
Background. The impact of multidrug-resistant organisms (MDROs), including non-fermentative bacilli (NFBs), is rising and underestimated, especially in intensive care units (ICUs). The growing prevalence of multidrug resistance (MDR) and extensive drug resistance (XDR) is challenging for clinicians, as the treatment options are limited.

Objectives. The purpose of this study was to analyze the extent of the epidemiological problem of multidrugresistant, extensively drug-resistant and pandrug-resistant (PDR) non-fermentative bacilli isolated from pneumonia and bloodstream infections (BSIs) in patients hospitalized in southern Poland.

Material and methods. This study included 253 NFBs belonging to Acinetobacter sp. (ACI), Pseudomonas sp. (PAR), and Stenotrophomonas sp. (STM). The microorganisms were identified, and susceptibility testing was performed using a semi-automatic system. The different patterns of resistance were defined as MDR, XDR, or PDR strains. Epidemiological typing of A. baumannii from ICUs was performed by repetitive polymerase chain reaction (rep- $P(R)$.

Results. More than half of the strains (57.7\%) were isolated within ICUs. ACl-strains came significantly more often from ICU wards. The highest prevalence of ACl and PAR was found in pneumonia, whereas STM dominated in BSIs. ACls were more frequently resistant than other pathogens to all studied antibiotics except colistin $(n=76 ; 58.9 \%)$, and they belonged to the XDR category. Diversilab demonstrated the presence of 2 dominant clones in the ACl group, both classified as European Clone 2 (EUII).

Conclusions. Our results indicate serious potential therapeutic problems related to high antibiotic resistance of $\mathrm{ACl}$ isolates. The stratification of drug resistance (MDR/XDR/PDR) may become an important tool for the assessment of public health epidemiology and microbiological hazards at the local, national, and international level. It allows clear presentation of the issues concerning the epidemiology of highly resistant bacilli, and the exchange of information between medical staff and local representatives of public health for the implementation of effective measures to reduce drug resistance.
\end{abstract}

Key words: pneumonia, multidrug resistance, non-fermentative bacilli, bloodstream infections 


\section{Introduction}

The impact of multidrug-resistant organisms (MDROs), including non-fermentative bacilli (NFBs), in intensive care units (ICUs) is rising and underestimated. ${ }^{1}$

Pseudomonas aeruginosa (PAR) shows an intrinsic resistance to many antibiotics and can horizontally acquire novel resistance genes. ${ }^{2}$ This may be one of the reasons for the increasing number of reports of multi-drug resistant (MDR) strains. ${ }^{3}$ In particular, nosocomial isolates of Acinetobacter and Pseudomonas may exhibit high rates of resistance to antimicrobials. Carbapenems are important agents for the treatment of severe infections caused by $P$. aeruginosa and $A$. baumannii. Carbapenem resistance poses a real threat to prognosis, because treatment options are limited. Carbapenems are one of the groups of antibiotics with enhanced activity, but these agents can also be inactivated by various mechanisms. ${ }^{4}$

Colistin is a key therapeutic option for the treatment of carbapenem-resistant $A$. baumannii and $P$. aeruginosa, alone or in combination with other agents, such as tigecycline, ampicillin-sulbactam, and carbapenems. Monotherapy is usually recommended for uncomplicated infections, while combination therapy is normally recommended for severe infections, such as bacteremia and pneumonia, although at least in some cases, the advantage of combination therapy remains a matter of debate. ${ }^{5}$ It is supposed that colistin resistance emerges under selective pressure in individual patients rather than through patient-to-patient transmission. ${ }^{6}$ A surveillance study of US hospitals revealed that $5.3 \%$ of all Acinetobacter strains were resistant to colistin. ${ }^{7}$

In recent decades, a substantial reduction in mortality rates in ICUs has been reported, but the antimicrobial resistance profile of microorganisms causing infections has significantly changed. Several factors may explain the rapid spread of MDROs in ICUs: new mutations, the selection of resistant strains, and poor antibiotic management. Most guidelines have different recommendations depending on the risk of the presence of MDROs. ${ }^{1,8}$

\section{Objectives}

Modern medicine requires clear and explicit criteria to describe the phenomena of public health, and one of the major problems of public health is microbial drug resistance. The aim of this study was to analyze the extent of the epidemiological problem of highly multidrug-resistant (MDR), extensively drug-resistant (XDR) and pandrug-resistant (PDR) non-fermentative bacilli isolated from pneumonia and bloodstream infections (BSIs) in patients hospitalized in southern Poland.

\section{Material and methods}

\section{Population description}

This laboratory-based, multicenter study comprised consecutive non-repetitive NFB isolates received from pneumonia and BSIs, from hospitalized and non-hospitalized patients throughout southern Poland (Małopolska and Silesia regions), collected between January 1, 2013 and December 31,2013. The study included patients from 12 hospitals, 3 long-term care and outpatient care facilities operated by the Chair of Microbiology at Jagiellonian University Medical College (Kraków, Poland), and 2 collaborative laboratories from the Silesia region. Pneumonia and BSI were diagnosed in accordance with the definitions of the European Center for Disease Prevention and Control (ECDC). ${ }^{9}$

A single strain was derived from the first sample collected in the case of the first episode of infection; during recurrent infection, a further strain was qualified for the study, provided that 14 days had passed since the first episode of infection, in the absence of clinical symptoms between the episodes. To confirm a diagnosis of BSI, at least 2 samples were taken. In the cases when a blood sample was taken to confirm a diagnosis of pneumonia, the bacterial isolate was treated as the cause of pneumonia (if lacking an isolate from bronchoalveolar lavage fluid).

Relevant information about the patients, such as age, sex, type of infection, and place of hospitalization was also collected. According to information gathered by the collaborating laboratories, patients were classified as one of the following: hospital patients; patients from ICUs ( $\mathrm{n}=146 ; 57.7 \%)$, the Department of Internal Medicine $(\mathrm{n}=63 ; 24.9 \%)$ or the pulmonary medicine ward $(\mathrm{n}=29$; $11.5 \%$ ); or outpatients, including residents of long-term care facilities (LTCFs), people with infections diagnosed by a physician, and those staying in nursing homes or receiving home care $(\mathrm{n}=15 ; 5.9 \%)$. The tested strains came from pneumonia ( $\mathrm{n}=197 ; 77.9 \%)$, BSIs $(\mathrm{n}=52 ; 20.5 \%)$ and meningoencephalitis $(n=4 ; 1.6 \%)$.

\section{Bacterial isolates}

In 2013, 2,763 samples were examined in the collaborating laboratories. Microbiological examinations were performed on blood (1,721 samples), pleural puncture fluid (124 samples), and tracheobronchial aspirates and bronchoalveolar lavage fluid, when available (918 samples).

Microorganisms were identified using a semi-automatic system (Phoenix; Becton-Dickinson, Warszawa, Poland), according to standard methods. Among the samples tested, 253 were positive for pathogens belonging to NFBs. The studied strains belonged to the following groups: 1) ACI (Acinetobacter baumannii, $\mathrm{n}=125$; Acinetobacter junii, $\mathrm{n}=1$; Acinetobacter radioresistens, $\mathrm{n}=1$; Acinetobacter lwoffii, $\mathrm{n}=1$; and Acinetobacter ursingii, $\mathrm{n}=1$ ); 
2) PAR (Pseudomonas aeruginosa, $\mathrm{n}=86$; and Pseudomonas putida, $\mathrm{n}=4$ ); or 3) Others (Stenotrophomonas maltophilia, $\mathrm{n}=26$; Achromobacter denitrificans, $\mathrm{n}=5$; Comamonas testosteroni, $\mathrm{n}=1$; Ochrobactrum anthropi, $\mathrm{n}=1$; and Alcaligenes faecalis, $\mathrm{n}=1$ ).

\section{Drug resistance}

Susceptibility testing was performed using a semi-automatic system (Phoenix NMIC/ID-204; Becton-Dickinson, Warszawa, Poland). Antimicrobial susceptibility was assessed according to the current European Committee on Antimicrobial Susceptibility Testing guidelines (EUCAST; clinical breakpoint tables v. 5.0), ${ }^{10}$ and the results were considered resistant (R) and susceptible (S). Resistant and intermediate strains were grouped together as drugresistant. For ampicillin-sulbactam, cefaperozone-sulbactam and tetracycline, antimicrobial susceptibility was assessed according to the Clinical Laboratory Standards Institute guidelines (breakpoints for ampicillin-sulbactam: $\mathrm{R}<11, \mathrm{I}=12-15, \mathrm{~S}>15$; for cefaperozone-sulbactam: $\mathrm{R}<15, \mathrm{I}=16-20, \mathrm{~S}>21$; for tetracycline: $\mathrm{R}<14, \mathrm{I}=15-20$, $\mathrm{S}>21$ ). For all strains, antimicrobial susceptibility testing for colistin was performed using the E-test strips (bioMérieux, Warszawa, Poland). Twenty antibiotics from 11 antimicrobial categories were tested: aminoglycosides, carbapenems, cephalosporins, cephalosporins and inhibitors, fluoroquinolones, folate pathway inhibitors, monobactams, penicillins, penicillins and $\beta$-lactamase inhibitors, polymyxins, and tetracyclines. For S. maltophilia (STM), only trimethoprim-sulfamethoxazole was tested, according to the EUCAST guidelines. Resistance for an antimicrobial category meant resistance to all antimicrobial agents in the category (according to the above description).
Different patterns of resistance were defined according to Magiorakos et al. with the following modifications: 1) bacteria not susceptible to at least 1 agent in 3 or more antimicrobial categories were considered to be MDR strains; 2) bacteria not susceptible to at least 1 agent in more than 1 or 2 antimicrobial categories were considered to be XDR strains; and 3) bacteria not susceptible to any agents in any antimicrobial categories were considered to be PDR strains. ${ }^{11}$

\section{DiversiLab typing}

Epidemiological typing of 101 A. baumannii strains from 2 ICUs (only) was performed by repetitive polymerase chain reaction (rep PCR) (DiversiLab System; bioMérieux, Warszawa, Poland) as previously described. ${ }^{4}$ Isolates that clustered $\geq 91.3 \%$ were considered related.

\section{Statistical methods}

The differences between 2 groups of patients with an infection caused by Acinetobacter spp. vs Pseudomonas spp. isolates were evaluated with an unpaired t-test (Welsh's t-test). Analysis of variance was used to compare multiple parameters, and their frequency was compared with the $X^{2}$ test or G-test (Table 1 ). The association between etiology and the type of infections, and the type of units, etc. was measured with an odds ratio (OR) and a 95\% confidence interval (CI). Factorial ANOVA model (logit link function and Poisson distribution of dependent variables) was used to check the influence of sex, age and type of ward on the number of antibiotic groups for which strains (ACI and PAR) were resistant (Table 4). The computer software package Statistica PL v. 6.0 was applied, and $\mathrm{p}<0.05$ was regarded as significant.

Table 1. Characteristics of patients with invasive infection caused by NFB isolates

\begin{tabular}{|c|c|c|c|c|c|}
\hline Origin of the bacterial strains evaluated in the study & $\begin{array}{l}\text { Acinetobacter spp. } \\
\qquad(\mathrm{n}=129)\end{array}$ & $\begin{array}{l}\text { Pseudomonas } \\
\text { spp. }(\mathrm{n}=90)\end{array}$ & $\begin{array}{l}\text { Others* } \\
(n=34)\end{array}$ & p-value & Statistical test \\
\hline Age of patients [years], mean $\pm S D$ & $60.6 \pm 17.2$ & $59.9 \pm 15.2$ & $63.7 \pm 19.1$ & 0.5291 & Student's t-test \\
\hline $\begin{array}{l}\text { Age of patients depending on the place of hospitalization } \\
\text { [years], mean } \pm S D \\
\text { Department of Internal Medicine }(n=63) \\
\text { ICU }(n=146) \\
\text { Pulmonary medicine }(n=29) \\
\text { outpatients with LTCF residents }(n=15)\end{array}$ & & $\begin{array}{c}67.5 \pm 16.6 \\
58.2 \pm 16.7 \\
61.9 \pm 7.6 \\
72.0 \pm 8.0\end{array}$ & & $<0.0001$ & Welsh's t-test \\
\hline Sex of patients, male ( $n$; \%) & $85 ; 65.9$ & $67 ; 74.4$ & $22 ; 64.7$ & 0.3378 & $x^{2}$ \\
\hline ICU patients (n; \%) & $101 ; 78.3$ & $36 ; 40.0$ & $9 ; 26.4$ & $<0.0001$ & $\mathrm{G}^{2}$ \\
\hline Meningoencephalitis (n; \%) & $4 ; 3.1$ & 0 & 0 & \multirow{3}{*}{$<0.0001$} & \multirow{3}{*}{$\mathrm{G}^{2}$} \\
\hline Pneumonia (n; \%) & $102 ; 79.0$ & $78 ; 86.7$ & $17 ; 50$ & & \\
\hline BSI (n; \%) & $23 ; 17.8$ & $1 ; 13.3$ & $17 ; 50$ & & \\
\hline
\end{tabular}

NFB - non-fermentative bacilli; ICU - intensive care unit; LTCF - long-term care facility; BSI - bloodstream infection; SD - standard deviation; * Others included: Stenotrophomonas maltophilia, $n=26$; Achromobacter denitrificans, $n=5 ;$ Comamonas testosteroni, $n=1 ;$ Ochrobactrum anthropi, $n=1 ;$ and Alcaligenes faecalis, $\mathrm{n}=1$. 


\section{Ethics}

The use of data collected for scientific purposes of this study was approved by the Bioethics Committee of Jagiellonian University Medical College (No. KBET/362/B/2012). All data entered into the electronic database and analyzed during this research were previously anonymized and de-identified.

\section{Results}

\section{Study population}

The average age of the study population was 60.8 years (SD: 16.3 years). There was a predominance of males ( $\mathrm{n}=174)$; however, there was no association between the occurrence of microbial species and the sex or age of patients (Table 1). The age of outpatients differed from the age of inpatients and LTCF patients; significantly older patients received home care or stayed in LTCFs, and significantly younger patients were treated in hospital (Table 1).

ACI strains came significantly more often from ICU wards, whereas PAR strains were from pulmonology wards, outpatients, and residents of LTCFs $\left(\mathrm{g}^{2}=52.762 ; \mathrm{p}<0.0001\right)$ (Table 1). The tested NFB strains appeared with different frequencies in different types of infections. The highest prevalence of ACI and PAR was found in pneumonia, and STM in BSIs (Table 2). Regardless of the type of unit, PAR strains were significantly less frequent in pneumonia than ACI (OR: 0.21; 95\% CI: 0.133-0.335), and in BSIs, strains from the Others group were significantly more frequently observed compared with other infections (OR: 4.74; 95\% CI: 2.208-10.19) (Table 2). Differentiating between ICU and non-ICU units, ACI strains were significantly more frequent in pneumonia in ICUs than other species (OR: 5.8; 95\% CI: 3.11-10.86) (Table 2). In the case of BSI, there were considerably more isolated strains from the Others group in non-ICU units than other species (OR: 25.3; 95\% CI: 4.75-134.89) (Table 2).

\section{Drug resistance}

More than $75 \%$ of ACI strains were resistant to 14 of 16 antimicrobials. Among ACI strains, the proportion of XDR strains was the highest among all 3 studied groups of pathogens ( $\mathrm{n}=99 ; 76.1 \%)$, and most of these isolates were resistant to all studied antibiotics with the exception of colistin ( $\mathrm{n}=76 ; 58.9 \%$ ) (Table 3 ). The minimal inhibitory concentration $\left(\mathrm{MIC}_{50}\right)$ for colistin in the ACI group was $1 \mathrm{mg} / \mathrm{L}$.

Two PAR strains (2.2\%) were resistant to all studied antibiotics with the exception of netilmicin and colistin. XDR PAR strains were less common than XDR ACI, 8.9\% vs 76.1\% (OR: 0.049; 95\% CI: 0.022-0.109). MDR strains occurred with a frequency of $28.9 \%(\mathrm{n}=26)$ (Table 3$)$. The $\mathrm{MIC}_{50}$ for colistin in the PAR group was $1 \mathrm{mg} / \mathrm{L}$.
Table 2. Frequency of isolation of NFB strains in various types of severe infection

\begin{tabular}{|c|c|c|c|c|}
\hline \multicolumn{2}{|c|}{$\begin{array}{c}\text { Infections caused } \\
\text { by NFB in hospital } \\
\text { patients }\end{array}$} & Meningitis & BSI & Pneumonia \\
\hline \multicolumn{5}{|c|}{$\mathrm{ACl}$} \\
\hline \multirow{3}{*}{ No. } & $\mathrm{ICU}$ & $3(75 \%)$ & 19 (82.6\%) & 79 (77.5\%) \\
\hline & non-ICU & $1(25 \%)$ & $4(17.4 \%)$ & $23(22.5 \%)$ \\
\hline & total & $4(100 \%)$ & $23(100 \%)$ & $102(100 \%)$ \\
\hline \multicolumn{2}{|c|}{ Prevalence $^{a}(\%)$} & 3.2 & 1.3 & 11.1 \\
\hline \multicolumn{5}{|c|}{ PAR } \\
\hline \multirow{3}{*}{ No. } & ICU & $0(0 \%)$ & 8 (66.7\%) & 28 (35.9\%) \\
\hline & non-ICU & $0(0 \%)$ & $4(33.3 \%)$ & $50(64.1 \%)$ \\
\hline & total & $0(0 \%)$ & $12(100 \%)$ & 78 (100\%) \\
\hline \multicolumn{2}{|c|}{ Prevalenceb (\%) } & 0.0 & 0.7 & 8.5 \\
\hline \multicolumn{5}{|c|}{ Others* } \\
\hline \multirow{3}{*}{ No. } & ICU & $0(0 \%)$ & $2(11.8 \%)$ & $7(41.2 \%)$ \\
\hline & non-ICU & $0(0 \%)$ & 15 (88.2\%) & $10(58.8 \%)$ \\
\hline & total & $0(0 \%)$ & $17(100 \%)$ & 17 (100\%) \\
\hline \multicolumn{2}{|c|}{ Prevalencec (\%) } & 0.0 & 1.0 & 1.9 \\
\hline
\end{tabular}

$\mathrm{ACl}$ - Acinetobacter baumannii; BSI - bloodstream infection;

ICU - intensive care units; NFB - non-fermentative bacilli;

PAR - Pseudomonas aeruginosa; * Others included: Stenotrophomonas maltophilia, $\mathrm{n}=26$; Achromobacter denitrificans, $\mathrm{n}=5$; Comamonas

testosteroni, $\mathrm{n}=1$; Ochrobactrum anthropi, $\mathrm{n}=1$; and Alcaligenes faecalis, $\mathrm{n}=1$;

calculated as follows:

(number of $\mathrm{ACl}$ meningitis/number of all cerebrospinal fluid cultures) $\times 100 \%$

(number of $\mathrm{ACI} \mathrm{BSI} /$ number of all blood cultures) $\times 100 \%$

(number of $\mathrm{ACl}$ pneumonia/number of all BAL cultures) $\times 100 \%$;

${ }^{b}$ calculated as follows:

(number of PAR meningitis/number of all cerebrospinal fluid cultures) $\times 100 \%$

(number of PAR BSI/number of all blood cultures) $\times 100 \%$

(number of PAR pneumonia/number of all BAL cultures) $\times 100 \%$; calculated as follows:

(number of OTHERS meningitis/number of all cerebrospinal fluid cultures) $\times 100 \%$

(number of OTHERS BSI/number of all blood cultures) $\times 100 \%$ (number of OTHERS pneumonia/number of all BAL cultures) $\times 100 \%$; $\mathrm{BAL}$ - bronchoalveolar lavage.

In strains from the Others group, 97\% were susceptible to sulphametoxazole-trimetoprim (SXT), and only 1 S. maltophilia - was resistant to SXT.

No PDR strains were identified in the studied group. Almost $60 \%$ of ACI strains were nearly PDR strains, and they were susceptible only to polymyxin antimicrobials.

\section{Factorial ANOVA analysis}

In the model of factorial analysis, the Poisson distribution of the dependent variable (effect) and the logit link function were assumed. The model showed a significant fit $\left(\chi^{2}=151.5078 ; \mathrm{df}=9 ; \mathrm{p}<0.0001\right)$. The analysis of parameters (Table 4) shows that the NFBs isolated from infections in ICUs were resistant to more antibiotic groups than 
Table 3. Resistance of the type of MDR to a selected group of antibiotics

\begin{tabular}{|c|c|c|}
\hline $\begin{array}{l}\text { Antimicrobial (sub-)classes and } \\
\text { antimicrobials used for testing }\end{array}$ & $\begin{array}{c}\text { Acinetobacter } \\
\text { spp. } \\
(n=129)\end{array}$ & $\begin{array}{c}\text { Pseudomonas } \\
\text { spp. } \\
(\mathrm{n}=90)\end{array}$ \\
\hline \multicolumn{3}{|c|}{ Aminoglycosides (\%) } \\
\hline gentamicin & 82.9 & 28.9 \\
\hline tobramycin & 81.4 & 23.3 \\
\hline amikacin & 86.0 & 30.0 \\
\hline netilmicin & 83.7 & 22.2 \\
\hline \multicolumn{3}{|c|}{ Antipseudomonal carbapenems (\%) } \\
\hline imipenem & 79.8 & 31.1 \\
\hline meropenem & 80.6 & 38.9 \\
\hline \multicolumn{3}{|c|}{ Extended-spectrum cephalosporins (\%) } \\
\hline ceftazidime & 92.2 & 25.6 \\
\hline cefepime & 92.2 & 28.9 \\
\hline \multicolumn{3}{|c|}{ Antipseudomonal fluoroquinolones (\%) } \\
\hline ciprofloxacin & 91.5 & 47.8 \\
\hline levofloxacin & 90.7 & 47.8 \\
\hline penicillins (piperacillin) (\%) & $\mathrm{n} / \mathrm{a}$ & 26.7 \\
\hline \multicolumn{3}{|c|}{ Penicillins and $\beta$-lactamase inhibitors (\%) } \\
\hline ticarcillin-clavulanic acid & $\mathrm{n} / \mathrm{a}$ & 28.9 \\
\hline piperacillin-tazobactam & 90.7 & 24.4 \\
\hline ampicillin-sulbactam & 77.5 & $\mathrm{n} / \mathrm{a}$ \\
\hline monobactams (\%) & $\mathrm{n} / \mathrm{a}$ & 96.7 \\
\hline folate pathway inhibitors (\%) & 91.5 & $\mathrm{n} / \mathrm{a}$ \\
\hline tetracyclines (\%) & 89.9 & $\mathrm{n} / \mathrm{a}$ \\
\hline cephalosporins and inhibitors (\%) & $\mathrm{n} / \mathrm{a}$ & 17.8 \\
\hline \multicolumn{3}{|c|}{ Polymyxins (\%) } \\
\hline colistin & 0.0 & 0.0 \\
\hline \multicolumn{3}{|c|}{ Type of resistance } \\
\hline sensitive (\%) & 5.4 & 27.8 \\
\hline MDR (\%) & 14.6 & 28.9 \\
\hline XDR (\%) & 76.1 & 8.9 \\
\hline PDR (\%) & 0.0 & 0.0 \\
\hline Other (\%) & 3.9 & 34.4 \\
\hline
\end{tabular}

MDR - multidrug-resistant; XDR - extensively drug-resistant;

PDR - pandrug-resistant; $n / a$ - not applicable. isolates from other wards. In addition, ACI organisms were resistant to more antibiotic groups than the PAR organisms (STM were not considered in the model).

\section{DiversiLab typing}

DiversiLab demonstrated the presence of 2 dominant clones in the ACI group. Clone 1 included 24 isolates and clone 2 included 55 isolates. Both of these clones were classified as European Clone 2 (EUII). Other listed clones (3-6) consisted of 2 strains. Twelve strains were distinguished as unique. Two isolates were non-typable. Most isolates belonging to clone 1 (67.8\%) and clone 2 (80\%) were susceptible only to colistin. Among the unique isolates, 30\% had a pattern of resistance to colistin alone.

\section{Discussion}

Non-fermentative Gram-negative bacilli have emerged as major agents of pneumonia (especially ventilator-associated), and their resistance to antibiotics, particularly to carbapenems, has become a therapeutic challenge.

Acinetobacter baumannii has become one of the most difficult nosocomial pathogens to control and treat, with a mortality rate of $\sim 30 \%,{ }^{12}$ and $P$. aeruginosa is a highly virulent organism with a mortality rate of $40-60 \% .{ }^{5} \mathrm{Aci}$ netobacter baumannii can also cause bloodstream, urinary tract, and wound infections. ${ }^{13}$

In our study, the highest prevalence of ACI and PAR was found in pneumonia, and STM was significantly more frequently observed in BSIs.

The most serious issue in the treatment of $A$. baumannii infection are XDR strains, because the number of active agents is limited. ${ }^{14}$ Our study also showed that the greatest problem was the extremely drug-resistant $A$. baumannii (76\%), compared with MDR strains (14.6\%).

The highest resistance among $A$. baumannii strains in our study was observed for aminoglycosides, cephalosporins, fluoroquinolones, carbapenems, and SXT. According to the European Antimicrobial Resistance Surveillance Network (EARS-Net), antibiotic resistance

Table 4. Independent predictors of antimicrobial resistance: Factorial ANOVA analysis

\begin{tabular}{|l|c|c|c|}
\hline & Estimate & Std Err & L-R $\chi^{2}$ \\
\hline Age of patients & 0.000262 & 0.002001 & 0.017154 \\
\hline Type of patient care: internal medicine & 0.140364 & 0.178104 & 0.703742 \\
\hline Type of patient care: intensive care units & 0.406582 & 0.167152 & 0.8958 \\
\hline Type of patient care: outpatients & -0.37352 & 0.304834 & 1.507221 \\
\hline Type of patient care: pulmonary medicine & -0.08715 & 0.1922 & 0.0026 \\
\hline Sex of patients: male & -0.03265 & 0.036124 & 0.192896 \\
\hline Studied non-fermentative bacilli: ACl & 0.322053 & 0.041334 & 0.822937 \\
\hline
\end{tabular}

$\mathrm{ACl}$ - Acinetobacter baumannii; Std Err - standard error; L-R - likelihood ratio. 
in Acinetobacter species shows large variations across Europe, with generally high percentages of resistance reported in southern Europe and lower percentages in northern Europe. Combined resistance to fluoroquinolones, aminoglycosides and carbapenems was $\geq 20 \%$ in 12 of the 23 countries reporting susceptibility results for 10 or more isolates. Carbapenem-resistant A. baumannii reached $>25 \%$ in 8 of the 18 countries reporting data. Resistance to polymyxins, a group of last-line antibiotics, was observed in 5\% of the isolates, mostly from southern Europe. $^{15}$

In 2010, the European Centre for Disease Prevention and Control (ECDC) reported that $\sim 15 \%$ of $P$. aeruginosa strains were MDR, in contrast to 1993, when the reported value was only $4 \% .{ }^{16}$ It is worrying to note that $1 / 3$ of the isolates were MDR, and $8.9 \%$ were XDR. Taken together, this means that $\sim 40 \%$ of the population are at risk of serious problems with the treatment of severe infections, such as BSI and pneumonia.

According to EARS-Net, $32.3 \%$ of the PAR isolates from invasive infections in 2013 were carbapenem-resistant, which is comparable to our results (about $1 / 3$ of the studied strains were resistant to imipenem). This is more than in previous reports from Poland, where only $10.5 \%$ of the isolates were carbapenem-resistant. ${ }^{17}$

We also noted a high number of PAR strains resistant to aminoglycosides ( $30 \%)$. Such a problem does not exist in countries such as Sweden (3\%) or Norway (1.5\%), but the situation is different in Greece (40\%) or the Czech Republic (25\%). ${ }^{15}$

The percentage of strains resistant to fluoroquinolones is also worrying, as about half of the tested strains were resistant. This rate is also higher than in Scandinavian countries, where $<10 \%$ of strains isolated from invasive infections are resistant to those antimicrobials. Furthermore, attention should be paid to the use of fluoroquinolones. Similar results were obtained in the case of urinary tract infections caused by PAR in Poland, which is understandable, as those antimicrobials are intended for use in urinary tract infections. ${ }^{18}$

Carbapenem-resistant strains are mostly susceptible to polymyxins; however, these antimicrobials are known to be toxic and are used as drugs of last resort. In our study, the greatest therapeutic problem was represented by the strains that were nearly pandrug-resistant and susceptible only to colistin. No strains resistant to colistin were reported in Poland.

It is of particular interest that, in our study, BSIs were frequently caused by bacteria from the OTHERS group, mainly STM. The World Health Organization (WHO) classified this pathogen as one of the leading MDROs in hospital settings. ${ }^{19}$ STM causes a wide range of infections, including respiratory tract infections and BSIs. Despite the large number of different global surveillance studies, there are still limited data on the prevalence and susceptibility patterns of STM. ${ }^{20}$ The worldwide prevalence of STM in BSIs was $\sim 0.8 \%$ in $2000-2004 .{ }^{21}$ It is difficult to assess how large the problem with STM was in our study population, because there were no data from other multicenter studies. However, reports from the German KISS program show a growing involvement of STM in the etiology of hospital infections, and the proportion of STM increased from 1.646 to 2.102 between 2001 and $2004 .^{21}$

There are limited antimicrobial options for infections due to STM because of its extensive resistance to most antibiotics. One drug recommended as a drug of choice is $\mathrm{SXT}^{22}$ Resistance rates may vary between different regions but, in general, they are $<10 \% .{ }^{23,24}$ One study from Poland on a set of 80 STM clinical isolates showed that only $71.3 \%$ of the strains were susceptible to SXT. ${ }^{25}$ Here, we detected only 1 resistant strain, which is not consistent with previous reports.

\section{Conclusions}

Our results indicate serious potential therapeutic problems related to the high antibiotic resistance of $A$. baumannii isolates. The stratification of drug resistance (MDR/XDR/PDR) may become an important tool for the assessment of public health epidemiology and microbiological hazards at the local, national, and international levels. It allows a clear presentation of the issues concerning the epidemiology of highly resistant bacilli, and the exchange of information between medical staff and local representatives of public health for the implementation of effective measures to reduce drug resistance. The growing prevalence of MDR and XDR is challenging for clinicians, because the treatment options are limited. New antimicrobial agents and treatment protocols are needed.

\section{References}

1. Martín-Loeches I, Diaz E, Vallés J. Risks for multidrug-resistant pathogens in the ICU. Curr Opin Crit Care. 2014;20(5):516-524.

2. Rossolini GM. Acquired metallo-beta-lactamases: An increasing clinical threat. Clin Infect Dis. 20051;41(11):1557-1558.

3. Obritsch MD, Fish DN, MacLaren R, Jung R. Nosocomial infections due to multidrug-resistant Pseudomonas aeruginosa: Epidemiology and treatment options. Pharmacotherapy. 2005;25:1353-1364.

4. Higgins PG, Dammhayn C, Hackel M, Seifert H. Global spread of carbapenem-resistant Acinetobacter baumannii. J Antimicrob Chemother. 2010;65(2):233-238. Erratum in:J Antimicrob Chemother. 2010;65(6):1317.

5. Rossolini GM, Mantengoli E. Treatment and control of severe infections caused by multiresistant Pseudomonas aeruginosa. Clin Microbiol Infect. 2005;11(4):17-32.

6. Qureshi ZA, Hittle LE, O'Hara JA, et al. Colistin-resistant Acinetobacter baumannii: Beyond carbapenem resistance. Clin Infect Dis. 2015;60(9):1295-1303.

7. O'Hara JA, Ambe LA, Casella LG, et al. Activities of vancomycin-containing regimens against colistin-resistant Acinetobacter baumannii clinical strains. Antimicrob Agents Chemother. 2013;57(5):21032108.

8. Vallés J, Martin-Loeches I, Torres A, et al. Epidemiology, antibiotic therapy and clinical outcomes of healthcare-associated pneumonia in critically ill patients: A Spanish cohort study. Intensive Care Med. 2014;40(4):572-581. 
9. European Centre for Disease Prevention and Control - An agency of the European Union. http://ecdc.europa.eu/en/publications/ Publications/healthcare-associated-infections-HAI-ICU-protocol. pdf Accessed August 2016.

10. EUCAST:Clinical breakpoints. http://www.eucast.org/clinical_breakpoints/ Accessed September 25, 2015

11. Magiorakos AP, Srinivasan A, Carey RB, et al. Multidrug resistant, extensively drug-resistant and pan-drug-resistant bacteria: An international expert proposal for interim standard definitions for acquired resistance. Clin Microbial Infect. 2012;18:268-281.

12. Perez F, Hujer AM, Hujer KM, Decker BK, Rather PN, Bonomo RA Global challenge of multidrug-resistant Acinetobacter baumannii. Antimicrob Agents Chemother. 2007;51(10):3471-384.

13. Antunes LC, Visca P, Towner KJ. Acinetobacter baumannii: Evolution of a global pathogen. Pathog Dis. 2014;71(3):292-301.

14. Maragakis LL, Perl TM. Acinetobacter baumannii: Epidemiology, antimicrobial resistance, and treatment options. Clin Infect Dis. 2008; 15;46(8):1254-1263. doi:10.1086/529198

15. Annual Epidemiological Report 2014. Antimicrobial resistance and healthcare-associated infections. http://ecdc.europa.eu/en/healthtopics/antimicrobial_resistance/database/Pages/database.aspx. Accessed August, 2015.

16. Obritsch MD, Fish DN, MacLaren R, Jung R. National surveillance of antimicrobial resistance in Pseudomonas aeruginosa isolates obtained from intensive care unit patients from 1993 to 2002. Antimicrob Agents Chemother. 2004;48(12):4606-4610.

17. Sacha $P$, Michalska A, Ojdana $D$, et al. Identification of plasmid OXA and other $\beta$-lactamase genes among carbapenem-resistant isolates of Pseudomonas aeruginosa from the Clinical University Hospital in north-eastern Poland. New Microbiol. 2015;38(2):271-275.

18. Pobiega M, Wojkowska-Mach J, Maciag J, et al. Virulence and antibi- otic resistance of Pseudomonas aeruginosa isolated from patients with urinary tract infections in southern Poland. Chemotherapy. 2014; 60(4):253-260.

19. Brooke JS. New strategies against Stenotrophomonas maltophilia: A serious worldwide intrinsically drug-resistant opportunistic pathogen. Expert Rev Anti Infect Ther. 2014;12(1):1-4.

20. Farrell DJ, Sader HS, Flamm RK, Jones RN. Ceftolozane/tazobactam activity tested against Gram-negative bacterial isolates from hospitalised patients with pneumonia in US and European medical centres (2012). Int J Antimicrob Agents. 2014; 43(6):533-539.

21. Meyer E, Schwab F, Gastmeier P, Rüden H, Daschner FD. Is the prevalence of Stenotrophomonas maltophilia isolation and nosocomial infection increasing in intensive care units? Eur J Clin Microbiol Infect Dis. 2006;25:711-714.

22. Wang $\mathrm{CH}$, Lin JC, Lin HA, et al. Comparisons between patients with trimethoprim-sulfamethoxazole-susceptible and trimethoprim-sulfamethoxazole-resistant Stenotrophomonas maltophilia monomicrobial bacteremia: A 10-year retrospective study. J Microbiol Immunol Infect. 2014;49(3):378-386.

23. Chung HS, Hong SG, Kim YR, et al. Antimicrobial susceptibility of Stenotrophomonas maltophilia isolates from Korea, and the activity of antimicrobial combinations against the isolates. J Korean Med Sci. 2013;28(1):62-66.

24. Livermore DM, Hope R, Brick G, Lillie M, Reynolds R; BSAC Working Parties on Resistance Surveillance. Non-susceptibility trends among Pseudomonas aeruginosa and other non-fermentative Gram-negative bacteria from bacteraemias in the UK and Ireland, 2001-06. J Antimicrob Chemother. 2008;62(2):ii55-63.

25. Hankiewicz-Ziołkowska K, Mikucka A, Gospodarek E. Clinical strains isolation and antibiotic susceptibility of Stenotrophomonas maltophilia. Med Dosw Mikrobiol. 2010;62(2):127-134. 